\title{
Estrogen receptor-negative breast carcinomas: a review of morphology and immunophenotypical analysis
}

\author{
Thomas C Putti ${ }^{1}$, Dalia M Abd El-Rehim ${ }^{2}$, Emad A Rakha ${ }^{2}$, Claire E Paish ${ }^{2}$, \\ Andrew HS Lee ${ }^{2}$, Sarah E Pinder ${ }^{2}$ and Ian O Ellis ${ }^{2}$ \\ ${ }^{1}$ Department of Pathology, National University of Singapore, Singapore and ${ }^{2}$ Department of Histopathology, \\ The Breast Unit, Nottingham City Hospital NHS Trust and University of Nottingham, UK
}

\begin{abstract}
Estrogen receptor (ER)-negative breast cancers are a group of tumors with poor prognosis and fewer cancer prevention and treatment strategies compared to ER-positive tumors. The aim of this study was to assess the morphological characteristics and immunohistochemical profile of ER-negative tumors and thus to understand the biological behavior and unique nature. In total, 291 consecutive ER-negative cases available from our primary breast cancer series were examined. Hematoxylin- and eosin-stained sections of all the cases were studied for several morphological parameters and their immunophenotype profile. These findings were correlated with patient and tumor characteristics and survival data. ER-negative tumors constituted $30 \%$ of the primary operable breast cancer series. The majority of tumors were grade $3(94 \%)$ and the commonest histological types were ductal/no specific type (85\%), and atypical medullary carcinoma (8\%). High-grade comedo-type necrosis, lymphoid stroma, central necrosis/fibrosis and pushing margins were the most common morphological features. The presence of a pushing margin showed a significant relation to androgen receptor negativity, absence of epidermal growth factor receptor expression and negative lymph nodes. Lymphoid stroma and comedo-necrosis correlated with higher tumor grade. ER-negative breast cancers are a distinct group of tumors with several common morphological features. Grade 3 histology, pushing margin, lymphoid stroma, comedo-type necrosis and central fibrosis/necrosis are the dominant morphological findings. The presence of a pushing margin appears to have a significant correlation with negative lymph node status. ER-negative tumors show a higher expression of p53, CerbB2 and epidermal growth factor receptor compared to ER-positive breast cancer. These unique features support the concept that ER-negative tumors are a morphologically and phenotypically distinct entity and provide a rationale for the study and use of newer promising agents in the treatment of ER-negative breast cancer.
\end{abstract}

Modern Pathology (2005) 18, 26-35, advance online publication, 27 August 2004; doi:10.1038/modpathol.3800255

Keywords: estrogen receptors; breast carcinoma; morphology; tissue microarray; immunohistochemistry

Breast cancer is a major concern worldwide and is responsible for one of the highest causes of death. Determination of estrogen receptor (ER) status on invasive carcinomas prior to therapeutic procedures has become a standard practice in the management of breast cancer and approximately $60-65 \%$ of primary breast cancers are ER-positive. ER has also proven to be a successful target for the treatment of ER-positive breast carcinomas; the effectiveness of antiestrogens such as tamoxifen and raloxifene is well known.

Correspondence: Professor IO Ellis, BMedSci, BM, BS, FRCPath, Department of Histopathology, Nottingham City Hospital NHS Trust, Hucknall Road, Nottingham NG5 1PB, UK.

E-mail: ian.ellis@nottingham.ac.uk

Received 16 April 2004; revised 7 July 2004; accepted 8 July 2004; published online 27 August 2004
Better-differentiated tumors are likely to be ERpositive and these ER-positive tumors have relatively better prognosis. ${ }^{1}$ Conversely, ER-negative tumors are more likely to be of higher histological grade, and the patients to have a decreased overall survival depending on age and lymph node status. ${ }^{2}$ ER receptor status of breast cancers in postmenopausal women is also associated with survival; a higher recurrence rate is observed in ER-negative group. ${ }^{3}$ Although most of the ER-negative tumors are presumed to be poorly differentiated, ${ }^{4}$ a significant proportion of a small subset of invasive cancers (adenoid cystic carcinoma, secretory carcinoma) are ER-negative. ${ }^{5,6}$ These tumors have an excellent prognosis with minimal regional recurrence. On the other hand, not all poorly differentiated, ER-negative tumors behave poorly. Medullary and 
atypical medullary cancers are reported in some series to have a relatively better prognosis than expected. ${ }^{7}$ Some ER-negative tumors also show a higher BRCA 1 germline mutation. ${ }^{8}$ All these features point towards the heterogeneous nature of an ER-negative subgroup of invasive breast cancers.

The purpose of this study was to determine the characteristics of ER-negative breast cancers through analysis of several morphological features and to correlate these features and their immunophenotypical profile with other prognostic variables and clinicopathological data to better understand their biological behavior.

\section{Materials and methods}

\section{Patients and tumors}

The study material was retrieved from a consecutive series of 1917 cases of primary operable invasive breast carcinoma obtained from the Nottingham Tenovus Primary Breast Carcinoma Series from patients presenting between 1986 and 1998. ER status was estimated immunohistochemically in 1805 of the tumors; $553(31 \%)$ cases were ERnegative while 1252 (69\%) carcinomas were ERpositive. From among those ER-negative tumors, 291 cases with retrievable histological material (blocks and slides) were included in this study. Patients' clinical history and cancer characteristics including tumor type,${ }^{9}$ histological grade,${ }^{10}$ tumor size, lymph node status and Nottingham Prognostic Index ${ }^{11}$ were obtained from the pathology database.

An hematoxylin- and eosin-stained section from a representative tumor block was examined for the various morphological parameters such as appearance of tumor margin, the presence of lymphoid stromal infiltrate (moderate to marked), clear cell changes, basaloid change, adenoid cystic pattern, acinic cell change, undifferentiated/small cell pattern, comedo-type necrosis, squamoid or spindle cell changes, presence of tumor giant cells and prominent central fibrosis/necrosis. The assessment was limited to the invasive portion of the tumor. A representative tissue microarray sample was stained to study the expression of various antigens by immunohistochemistry. The antibodies utilized included ER, androgen receptor (AR), cytokeratin 5/6 (CK5), cytokeratin 18 (CK18), smooth muscle actin, gross cystic disease fluid protein-15 (GCDFP), epidermal growth factor receptor (EGFR), BRCA1, CerbB2, p53 and E-cadherin (Table 1). ER was included to confirm the earlier findings on full section immunohistochemistry. As ER-negative tumors are generally associated with a poor prognosis, markers with putative prognostic value (EGFR, CerbB2, p53, BRCA1) were also included. GCDPF and smooth muscle actin were utilized to identify apocrine and salivary-type differentiation, and AR and E-cadherin to assess their general prevalence in ER-negative tumors. CK5 and CK18, which identify
Table 1 Primary antibodies

\begin{tabular}{llll}
\hline Antibody & Source & Clone & Dilution \\
\hline E-cadherin & Zymed & HECD-1 & $1: 200$ \\
p53 & Novocastra & DO7 & $1: 50$ \\
ER & Dako & 1D5 & $1: 80$ \\
Actin & Dako & 1A4 & $1: 2000$ \\
CerbB2 & Dako & A0485 & $1: 250$ \\
AR & Biogenex & F39.4.1 & $1: 30$ \\
CK5/6 & Novocastra & DS/16B4 & $1: 100$ \\
GCDFP & Novocastra & 23A3 & $1: 30$ \\
EGFR & Novocastra & DC10 & $1: 15$ \\
CK18 & Dako & MS110 & $1: 50$ \\
BRCA-1 & Oncogene & & \\
\hline
\end{tabular}

basal and luminal epithelial differentiation respectively, were also included.

\section{Tissue Arrays and Immunohistochemistry}

Tumor samples were arrayed as previously described. ${ }^{12}$ In brief, tissue cores with a diameter of $0.6 \mathrm{~mm}$ were punched from the representative tumor regions of each donor block. Each case was sampled twice from the center and the periphery of the tumor. Cores were precisely arrayed into a new recipient paraffin blocks using a tissue microarrayer (Beecher Instruments). Immunohistochemical staining was performed on $4 \mu \mathrm{m}$ thick sections using the avidin-biotin complex method. Briefly, tissue slides were deparaffinized with xylene and then rehydrated through three changes of alcohol. Endogenous peroxidase activity was blocked by incubation in a $0.3 \%$ hydrogen peroxide/methanol buffer. Antigen retrieval was carried out by microwave treatment of the slides in sodium citrate buffer $(\mathrm{pH}$ 6.0) for $20 \mathrm{~min}$. The slides were then rinsed in Trisbuffered saline (TBS) (pH 7.6) and incubated with normal swine serum in TBS $(1: 5)$ to block nonspecific staining. The slides were then incubated for $1 \mathrm{~h}$ with the primary antibody. After washing with TBS, sections were incubated with the secondary antibody (biotinylated goat anti-Mouse/Rabbit immunoglobulin; Duet K 0492, DakoCytomation, Denmark) (1:100) for $30 \mathrm{~min}$ then the avidinbiotin complex (1:100) for a further $45 \mathrm{~min}$. 3-3'Diaminobenzidine tetrahydrochloride (Dako liquid DAB plus, K3468, Denmark) was used as a chromogen and sections were counterstained with Mayer's hematoxylin. The negative controls were omission of the primary antibody.

\section{Evaluation of Immunohistochemical Staining}

Positive controls and internal areas of normal breast tissues showed strong and uniform staining of the epithelial cells lining the ducts and lobules with minimal back ground reactivity for various cytokeratins, smooth muscle actin, BRCA-1, E-cadherin and 
GCDFP. For evaluating EGFR, CerbB2 and p53 immunostaining, a known positive external control was utilized. Two cores were evaluated from each tumor. Each core was scored individually then the mean of the two readings was calculated. If one core was uninformative, the overall score applied was that of the remaining core. Assessment of staining was based on a semiquantitative approach. A modified histochemical score (H-score) was used which includes an assessment of both the intensity of staining and the percentage of stained cells. ${ }^{13}$ For the intensity, a score index of $0,1,2$ and 3 corresponding to negative, weak, moderate and strong staining intensity was used and the percentage of positive cells at each intensity was estimated subjectively. A final score of $0-300$ is the product of both the intensity and the percentage. Staining of ER and p53 was evaluated in the nuclei of the malignant cells and scored as positive or negative. An $H$-score of 0 and 50 were considered as cutoff points for positive staining of ER and rest of the markers, respectively. One observer scored the staining pattern (DAR), without previous knowledge of the outcomes on two separate occasions and a good correlation between the results was found.

\section{Statistical Analysis}

Statistical analysis was performed using SPSS 10.0 statistical software. Univariate and multivariate analysis were performed by $\chi^{2}$-test, log rank and Cox regression analysis, respectively. Nonparametric method methods were used for non-normally distributed data (including Mann-Whitney $U$ - and Kruskall-Wallis $H$-tests). Correlations between different markers were carried out using Pearson's correlation coefficient. Survival curves were analyzed by the method of Kaplan-Meier (Kaplan and Meier, 1958). A $P$-value $<0.05$ was considered significant.

\section{Results}

\section{Demographics of the Patients and Tumor Characteristics (Table 2)}

All the patients were female with an age range of 25-70 years (median 50.5 years). Among the cases with known menopausal status, 85 and 78 were premenopausal and postmenopausal, respectively. The commonest histological types were ductal/no specific type (NST), comprising $85 \%$ of the total cases and atypical medullary carcinoma (8\%).

Most of the tumors were more than $2 \mathrm{~cm}$ in size $(53 \%)$. Of the patients with known lymph node status, $148(51 \%)$ were lymph node metastases negative, while $96(33 \%)$ were stage 2 (one to three nodes positive) and 46 (16\%) stage 3 (four or more nodes positive). In comparison, only $36 \%$ of the patients in the entire series of both ER-positive and
Table 2 Patient characteristics and ER-negative tumor parameters

\begin{tabular}{lc}
\hline Histological tumor type & Number of cases (\%) \\
\hline No specific type (NST) & $246(85 \%)$ \\
Tubular mixed & $7(2.5 \%)$ \\
Typical medullary & $2(0.7 \%)$ \\
Atypical medullary & $24(8 \%)$ \\
Classic lobular & $4(1.4 \%)$ \\
Lobular mixed & $1(0.3 \%)$ \\
NST \& lobular mixed & $1(0.3 \%)$ \\
Mixed NST and special type & $1(0.3 \%)$ \\
Miscellaneous & $5(2 \%)$ \\
Age & $($ median 50.5 years) \\
& \\
Menopausal status & \\
Pre & $85(29 \%)$ \\
Post & $78(27 \%)$ \\
Unknown & 128 \\
Tumor size (cm) & \\
$<2$ & $136(47 \%)$ \\
$>2$ & $155(53 \%)$ \\
Nodal status & \\
Stage 1 (node negative) & \\
Stage 2 (1-3 nodes positive) & $148(51 \%)$ \\
Stage 3 (4 or more nodes & $96(33 \%)$ \\
positive) & $46(16 \%)$ \\
Grade & \\
1 & \\
2 & \\
3 & $03(1 \%)$ \\
Vascular invasion & $272(94 \%)$ \\
\hline & \\
\hline & \\
&
\end{tabular}

negative tumors, had lymph node metastases $(29 \%$ with 1-3 positive nodes and $8 \%$ with 4 or more positive nodes). A high proportion of the tumors were grade $3(94 \%)$ with a few grade 1 cases $(1 \%)$. In contrast, grade 3 tumors comprised less than half $(47.7 \%)$ of the entire primary breast cancer series. Definite vascular invasion was seen in 104 tumors (36\%). The Nottingham prognostic index in these cases ranged from 2.5 to 8.4 (mean 5.0).

\section{Morphological Features of Tumors (Figure 1 and Table 3)}

The presence of comedo-type tumor necrosis, lymphoid stroma, tumor giant cells, clear cell change, pushing margin and central necrosis/fibrosis were the most common additional morphological features seen in these tumors. Central necrosis/ fibrosis was seen in 22 cases $(8 \%)$.

\section{Immunohistochemical Stains on Tissue Microarray (Figure 2 and Table 4)}

Detectable nuclear immunoreactivity for ER was not seen in any case, confirming the earlier findings on full section immunohistochemistry. E-cadherin expression was seen in 253 cases (89\%), with weak 

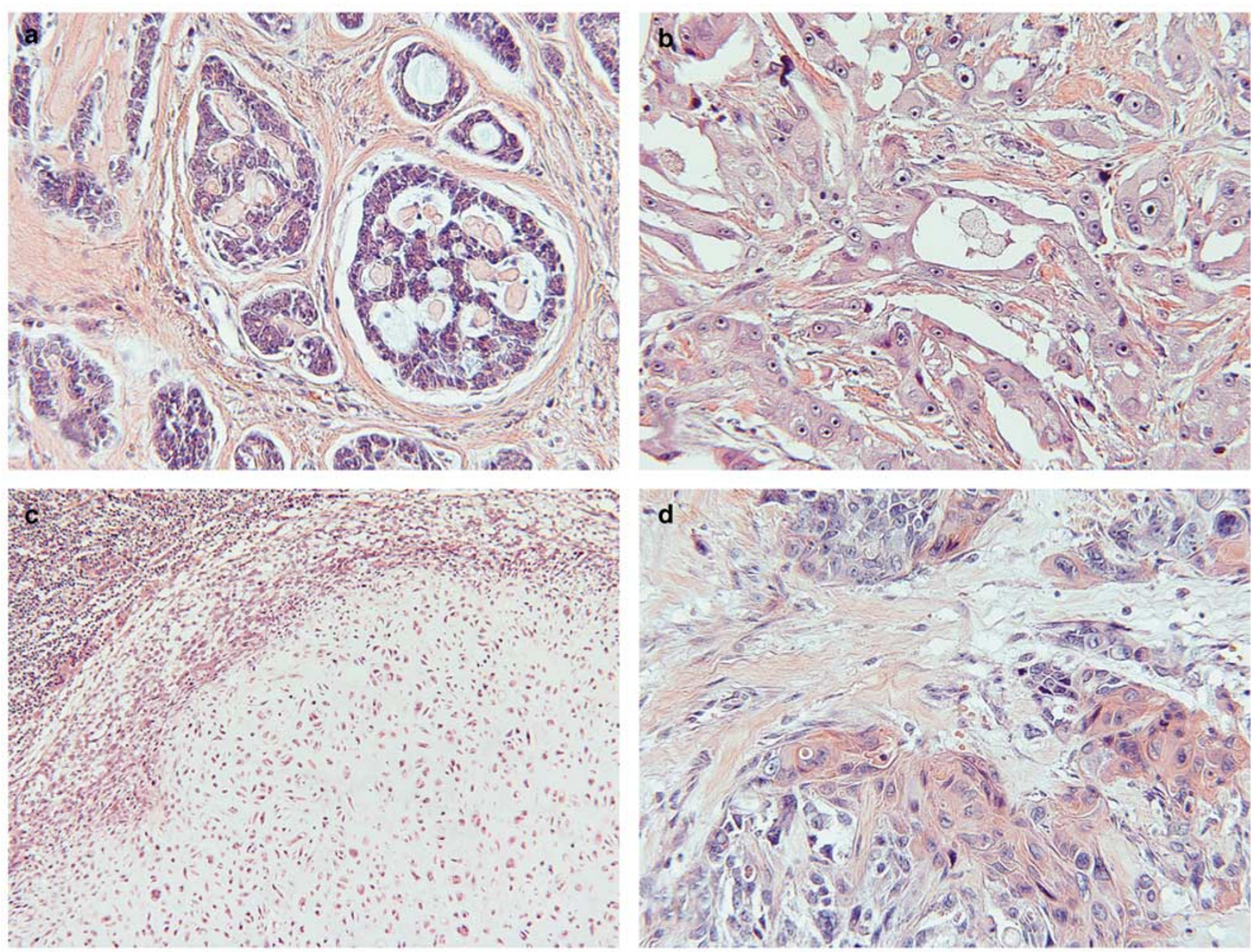

Figure 1 Some examples of ER-negative subset tumors and their morphological features: (a) Adenoid cystic carcinoma; (b) apocrine carcinoma; (c) metaplastic carcinoma with predominant cartilaginous matrix; (d) squamoid change; (e) clear cell change; (f) central necrosis; (g) marked lymphoid stromal infiltrate; (h) pushing tumor margin with dense inflammatory infiltrate at the periphery.

Table 3 Morphologic characteristics in ER-negative invasive breast carcinomas

Morphologic characteristic

Number of cases (\%)

Pushing margin

Lymphoid stroma

Clear cell changes

Basaloid cell change

Adenoid cystic pattern

Acinic cell change

Undifferentiated/small cells

Comedo-type necrosis

Squamoid change

Spindle cell change

Tumor giant cells

Central necrosis/fibrosis

$23(8 \%)$

$92(32 \%)$

$35(12 \%)$

$04(1 \%)$

$02(1 \%)$

$02(1 \%)$

$05(2 \%)$

$95(33 \%)$

$18(6 \%)$

$08(3 \%)$

$92(32 \%)$

$22(8 \%)$

expression in 129 (45\%) of them. CK18 was present in 193 tumors (69\%), p53 in 171 (60\%), and BRCA1 in $168(68 \%)$. CerbB2 was overexpressed in 91 $(32 \%)$ and EGFR in 108 tumors (41\%).

\section{Lymph Node Status, Survival Data and Tumor Morphology (Table 5)}

When the predominant tumor morphological features were compared to lymph node status, the presence of a pushing margin to the tumor showed a significant correlation with the absence of lymph node metastases. The rest of the morphological features were seen just as frequently in both nodepositive and -negative groups.

\section{Multivariate Analysis Including Morphological Features with Other Prognostic Factors (Table 6)}

The presence of a pushing margin showed a significant correlation with AR negativity, EGFR negativity, lymph node stage 1 disease, the absence of vascular invasion, and premenopausal status. The presence of a lymphoid stroma displayed a positive correlation with negative GCDFP and grade 3 lesions. The presence of clear cell change showed no significance with other parameters. The absence 

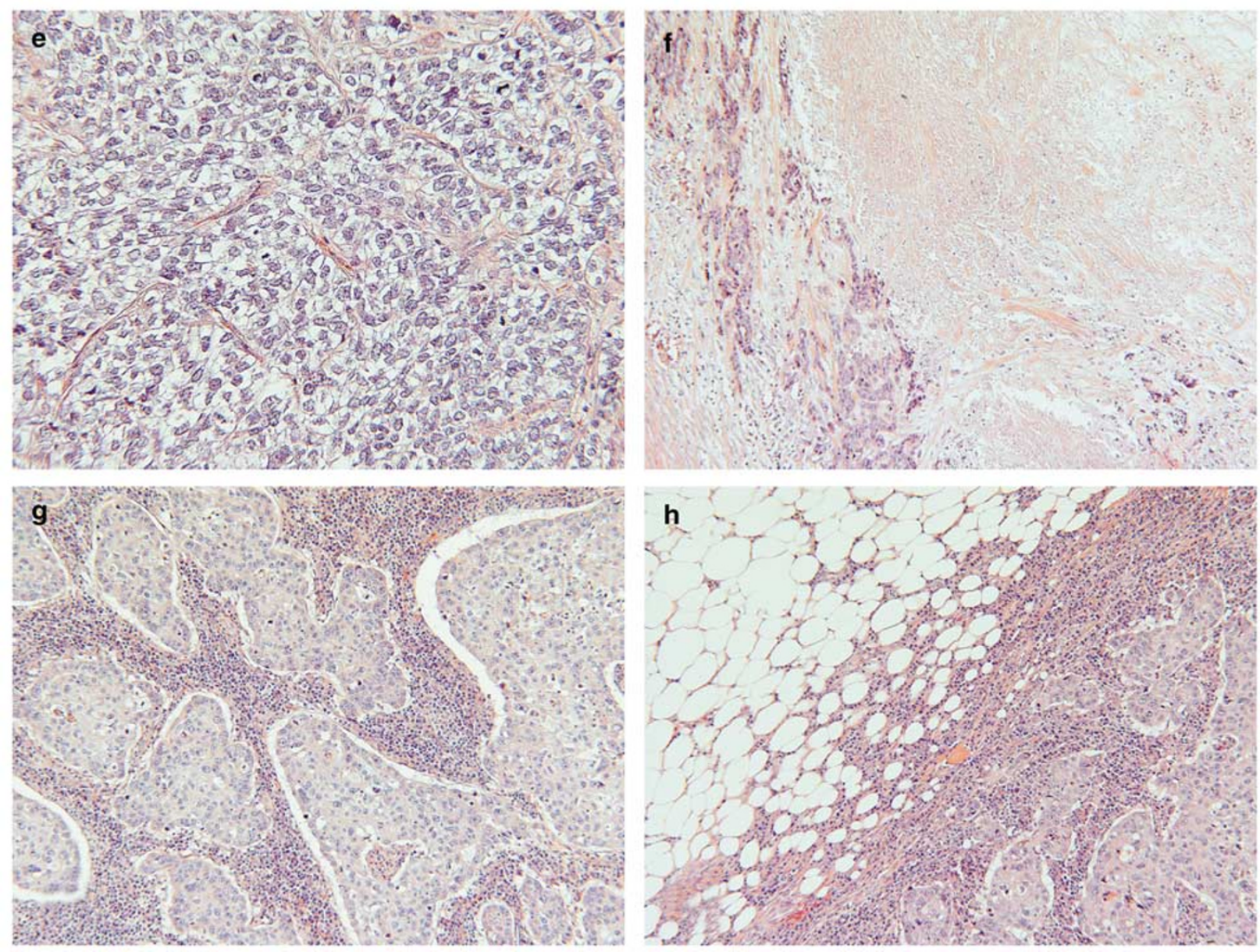

Figure 1 Continued.

of basaloid change correlated with grade 3 tumors. Both cases of adenoid cystic carcinoma show smooth muscle actin positivity and were of histological grade 1. Comedo-type necrosis correlated with AR negativity, CK5 staining, grade 3 tumors and greater tumor size.

\section{Discussion}

Breast cancer is a major concern worldwide and is one of the leading causes of death in women. The role of hormone receptors as a prognostic and therapeutic tool is widely accepted and estrogen receptor has proven to be a successful target for all ER-positive breast carcinomas. The overall mortality due to breast cancer has also shown a decline in the Western world in the recent years, attributed in part to the early application of various treatment modalities. ${ }^{14}$ In order to further reduce the mortality from breast cancer there is a desire to further examine and characterize the subset of ER-negative tumors, which are traditionally of poor prognosis and lack effective chemopreventive strategies.
There is scant information in the literature regarding the morphology and immunoprofile of ER-negative breast tumors. One of the best earlier studies on the prognostic strength of ER was the NSABP B-06 study, in which women with early stage breast cancer received no adjuvant systemic therapy ${ }^{15}$ Patients with ER-positive tumors had 5 -year disease-free survival (DFS) rates of $74 \%$ and 5 -year overall survival (OS) rates of $92 \%$. Conversely, patients with ER-negative tumors had 5-year DFS and OS of 66 and $82 \%$, respectively.

The majority of cases in this study were originally classified as ductal/no-specific type, followed by a high percentage of atypical medullary cases. Some of the cases diagnosed as no specific type were subsequently recategorized in the present study into other subtypes such as apocrine, metaplastic, small cell undifferentiated, solid lobular, papillary, secretory, and salivary gland-like tumors. The detailed morphology and immunophenotypical profile of these lesions will be discussed in a subsequent publication.

ER-negative invasive breast cancers include a variety of histological types and among them it is well recognized that the poorly differentiated 

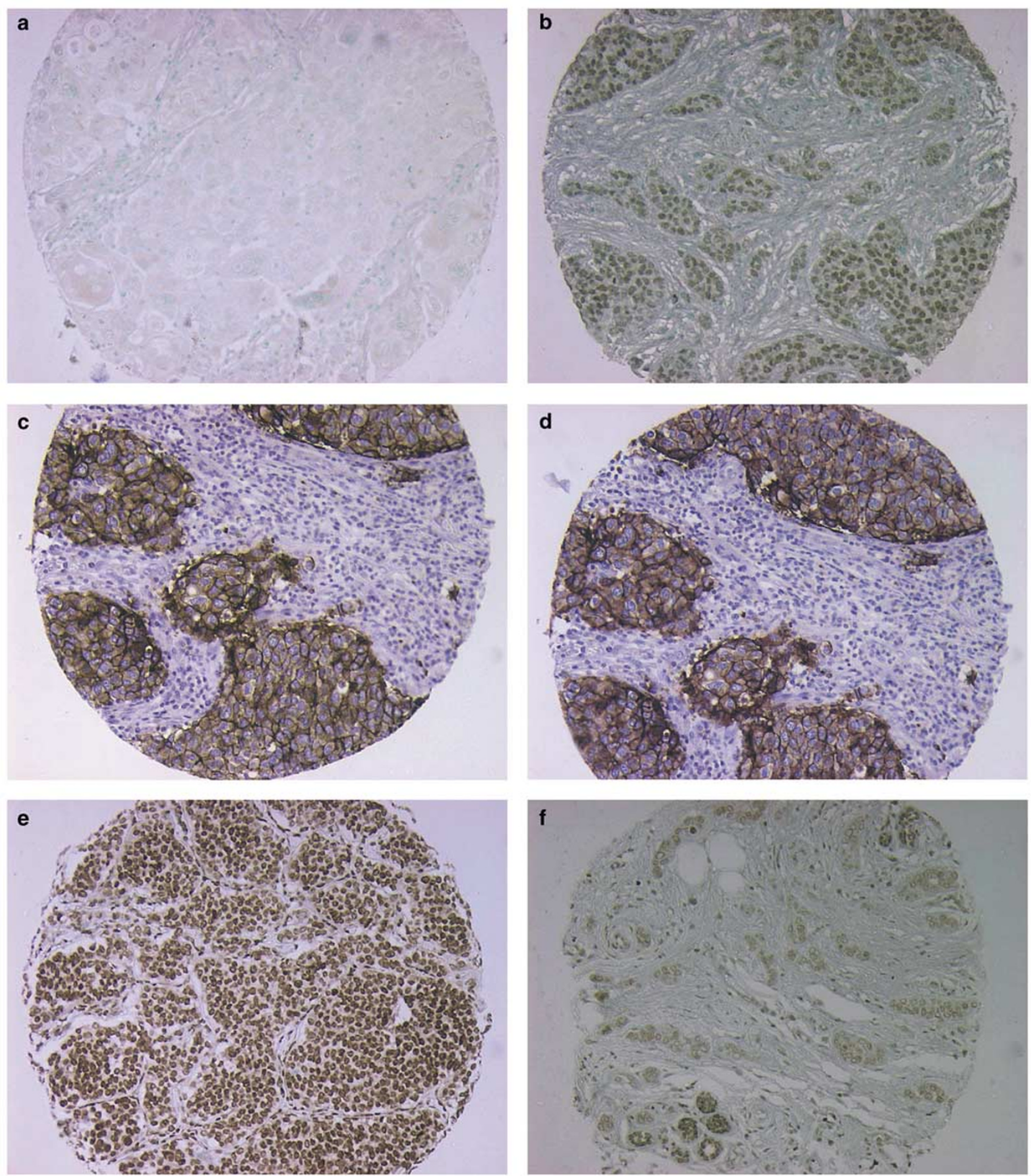

Figure 2 Few examples of immunophenotying studies on tissue microarray: (a) Negative immunostain for estrogen receptor; (b) androgen receptor-nuclear positivity; (c) EGFR—membrane stain; (d) CerbB2—strong membrane positivity; (e) BRCA1一positive in lobular carcinoma; (f) BRCA1—negative ductal/no specific type carcinoma with internal positive control.

medullary type of breast tumor shows a high rate of ER negativity. ${ }^{9}$ On the contrary, a few of the welldifferentiated breast carcinomas are also ER-negative. ${ }^{5,6}$ In our series, we have identified two ERnegative adenoid cystic carcinomas; both of them were of histological grade 1 with positivity for smooth muscle actin, highlighting their nonluminal epithelial phenotype. Breast and salivary glands share several morphological features, both being tubulo-acinar exocrine glands. Salivary gland-like lesions of breast are divided into two main groups, one with myoepithelial differentiation and the 
Table 4 Results of immunohistochemical analysis

\begin{tabular}{lrrc}
\hline Antibody & Negative & Positive & Valid cases (n) \\
\hline p53 & 115 & $171(60 \%)$ & 286 \\
AR & 205 & $66(24 \%)$ & 271 \\
BRCA1 & 78 & $168(68 \%)$ & 246 \\
EGFR & 153 & $108(41 \%)$ & 261 \\
CerbB2 & 198 & $91(32 \%)$ & 289 \\
GCDFP & 230 & $55(19 \%)$ & 285 \\
E-cadherin & 33 & $253(89 \%)$ & 286 \\
CK18 & 87 & $193(69 \%)$ & 280 \\
CK5/6 & 160 & $129(45 \%)$ & 289 \\
Actin & 211 & $75(26 \%)$ & 286 \\
\hline
\end{tabular}

Weak and strong expression were combined for BRCA, CerbB2 and E-cadherin.

other devoid of it. ${ }^{16}$ Adenoid cystic carcinomas belong to the former category and are typically ER-negative and are also known to overexpress KIT. ${ }^{5,17}$ The lack of ER expression in this form of grade 1 invasive breast cancer highlights the variation in the subgroup of ER-negative tumors; some tumors lacking ER in association with poor differentiation but others due to a nonluminal epithelial lineage.

When the dominant morphological features were correlated to axillary lymph node status, only the presence of a pushing margin showed a significant correlation to negative lymph node status (Table 5). This finding can be explained by the presence of a significantly higher number of medullary and atypical medullary tumors which tend to have a lower overall frequency of axillary lymph node metastases than patients with ductal/NST carcinomas. The other characteristics identified had almost equal distribution in relation to positive and negative node status. Scawn and Shousha, ${ }^{18}$ in a similar study of ER-negative invasive cancers, examined several of the morphological features assessed in the present study. They reported lymph node metastasis in $46 \%$ of their cases and a wide range of tumor sizes. Based on their findings they concluded that ER status of the tumors was determined early in its natural history and supported the existence of two divergent pathways for the development of ER-negative and positive tumors. In the present study, a similar proportion of ER-negative tumors had axillary lymph node disease $(49 \%)$, a higher proportion than in the series as a whole $(36 \%)$ and higher than the general incidence
Table 6 Summary of multivariate analysis of ER-negative tumors

\begin{tabular}{lll}
\hline Morphological feature & Prognostic factor & P-value \\
\hline Pushing margin & Negative AR & 0.043 \\
& Negative EGFR & 0.013 \\
& Lymph node stage 1 & 0.019 \\
& Absence of vascular invasion & 0.054 \\
& Premenopausal status & 0.005 \\
Lymphoid stroma & Negative GCDCP & 0.006 \\
& Grade 3 tumor & 0.012 \\
Comedo-type necrosis & Negative AR & 0.007 \\
& Positive CK5 & 0.011 \\
Tumor giant cells & Grade 3 tumor & 0.04 \\
Squamoid differentiation & Greater tumor size & 0.09 \\
Spindle cell change & Negative P53 & 0.03 \\
Basaloid change & Negative AR & 0.018 \\
& Negative E-cadherin & 0.04 \\
& Lower grade tumors & 0.003 \\
\end{tabular}

of $20-30 \%$ commonly observed in the breast cancer. ${ }^{19}$

The presence of prominent lymphoid infiltration in association with ER-negative tumors is supported by the hierarchical cluster analysis of ER-positive and -negative groups. ${ }^{20}$ Iwao et al examined a series of cases by gene-expression profiling and identified that immunoglobulin genes are primarily expressed in ER-negative groups, supporting B-lymphoid cell infiltration into these tumors. Data from the present study shows a prominent lymphoid stroma in approximately half of the cases, a feature showing a significant correlation to grade 3 carcinomas. The presence of lymphoid infiltrate in the tumor has been correlated with higher histological grade previously, but the prognostic significance is controversial with conflicting results. ${ }^{21}$ Most of the infiltrate is composed of lymphoid cells and macrophages with a variable admixture of plasma cells and eosinophils. A predominance of plasma cells is seen in medullary and atypical medullary tumors. Tumors with a marked lymphocytic reaction are reported almost always ER-negative. ${ }^{22}$ Subset analysis of the influence of lymphoplasmacytic reaction on prognosis has shown that it is related to nodal status, tumor grade and overexpression of Her2/neu oncogene. ${ }^{23}$

Several studies have reported an adverse effect of necrosis on clinical outcome. ${ }^{24-27}$ Necrosis in carcinoma has been associated with a higher incidence of axillary node metastases and a higher mortality

Table 5 Relationship between select tumor morphology and lymph node status

\begin{tabular}{lcccc}
\hline & Comedo-type necrosis no. & Lymphoid stroma no. & Tumor giant cells no. & Pushing margin no. \\
\hline LN positive & $44(46 \%)$ & $45(49 \%)$ & $46(51 \%)$ & $05(22 \%)$ \\
LN negative & $51(54 \%)$ & $46(51 \%)$ & $45(49 \%)$ & $18(78 \%)$ \\
Total & 95 & 91 & 91 & 23 \\
\hline
\end{tabular}

(no.)—number of cases; LN—lymph node. 
rate. ${ }^{24}$ Confluent tumor necrosis of any dimension has also been reported to be an independent predictor for early recurrence and death from disease. ${ }^{25}$ Gilchrist et al, however, indicated that the prognostic significance was time dependent, the effect manifested only in the first 2 years of the follow-up and beyond 10 years, it was no longer a significant prognostic factor for patients who remained disease free. A distinct entity 'centrally necrotizing carcinoma' was studied by Jiminez et $a l^{26}$ This was characterized by early development of systemic metastases and accelerated clinical course. These tumors displayed minimal tubule formation and a majority of them (94\%) were ER-negative. These studies support the view that extensive tumor necrosis is an unfavorable prognostic factor and may be related to the rapid growth of the tumor, exceeding angiogenesis to a substantial degree. One-third of the cases in the present series displayed comedo-type necrosis, and a significant proportion of them also had extensive central necrosis/fibrosis. The presence of comedo-type necrosis correlated with AR negativity, higher grade and greater size but no significant correlation was noted with lymph node metastases. The presence of a fibrotic/necrotic focus in the centre of the tumor has been reported to be an adverse prognostic factor. ${ }^{28,29}$ It has been suggested that the finding of a fibrotic focus is a surrogate marker for hypoxiadriven ongoing angiogenesis and has been reported to be associated with a higher relapse rate and worse overall prognosis. The rest of the morphological features assessed, including the presence of clear cell differentiation, squamoid and spindle cell change did not show significant correlation to any of the other parameters studied. This could be due to low number of events seen in our series, but may reflect that these are not important characteristics with regard to prognosis.

The presence of vascular invasion and increasing tumor grade has been shown to be an independent prognostic factor. ${ }^{30,31}$ The presence of vascular invasion was associated with larger tumors, grade 3 disease, lymph node metastases and ductal/no specific type carcinomas. ${ }^{30}$ Increasing tumor grade is related to increased local recurrence after conserving surgery, increased size and ER-negativity. ${ }^{31}$ In the present series, definite vascular invasion and histological grade 3 tumors were present in 36 and $94 \%$ of cases, respectively. The absence of a pushing margin correlated with the presence of vascular invasion $(P=0.019)$. A statistical correlation between the presence of lymphoid stroma and comedotype necrosis and histological grade 3 lesions was also seen.

A study on AR expression in ER-negative breast cancers found that AR expression was correlated with longer survival in a cohort of women with ERnegative tumors. ${ }^{32}$ The data from Agoff et al suggested that AR status could be used to subdivide ER-negative tumors into more and less favorable prognostic groups. Sixty-six (24\%) of the ERnegative tumors in our study expressed AR, a feature associated with a nonpushing margin and the absence of comedo-type necrosis. AR expression in apocrine lesions has been reported to show a distinct pattern of overexpression, accompanied by the loss of estrogen and progesterone receptors. ${ }^{33}$ GCDFP expression was seen in a small percentage of our cases $(19 \%)$ in the present series, the majority of them $(66 \%)$ also showed AR positivity, signifying that $\mathrm{AR}$ expression is frequent in lesions with apocrine differentiation.

Elevated EGFR expression, in the absence of gene amplification is associated with estrogen receptor negativity. ${ }^{34}$ EGFR was overexpressed in 108 (41\%) of our cases and expression showed significant association with absence of pushing margin and presence of tumor giant cells. Many promising agents for the prevention of ER-negative tumors are on trial. An orally active EGFR inhibitor that blocks signal transduction processes in the proliferation and survival of breast cancer cells was examined in MMTV-erbB2 transgenic mice. ${ }^{35}$ Lu et al showed a reduced proliferation of tumor cells and increased expression of cell cycle regulator p27, providing a rationale for use of these agents in prevention of ERnegative breast cancer.

CerbB2 expression was inversely correlated with ER expression. ${ }^{36}$ In the present study, CerbB2 positivity was seen in almost $45 \%$ of ER-negative cases, although the overall expression is around $30 \%$ in the entire ER-positive and -negative series and in series reported by others. ${ }^{37-39}$ Similarly, a higher percentage of ER-negative tumors in the present study also showed aberrant expression of p53 $(60 \%)$. A strong association between specific mutations and short survival and poor response to treatment has been reported; a meta-analysis study of somatic mutations of p53 in breast cancers reported overall relative hazards for recurrence and death at 1.5 and 2.0, respectively. ${ }^{40}$ Previous studies have found an inverse association between luminal cytokeratin expression (CK18) and tumor grade, recurrence rate and ER status. ${ }^{41}$ The results of our study with decreased expression of luminal markers and increased expression of basal markers also correlates with poor prognostic features and behavior of ER-negative tumors. ${ }^{42}$

E-cadherin is an important mediator of cell-to-cell interaction and has been reported to be aberrantly expressed or absent in most of the lobular cancers of breast. ${ }^{43}$ In contrast, its expression is aberrant or lost in only a minority of ductal cancers. In all, 11 and $45 \%$ of our ER-negative cancers showed E-cadherin loss and aberrant expression, respectively. These data are in keeping with the predominance of grade 3 ductal carcinomas and few invasive lobular carcinomas in this series. There appears to be slightly higher proportion of E-cadherin aberrations in ER-negative tumors. In a study of E-cadherin expression in primary carcinomas and its distant 
metastases, the authors concluded that aberrant expression of E-cadherin is frequent in invasive ductal carcinomas that progress to develop distant metastases. ${ }^{44}$ However, the connection between ER and E-cadherin is complex. Oesterreich et $a l^{45}$ showed that ER-negative cell lines often showed an absence of E-cadherin, while in ER-positive tumors, estrogen could cause downregulation of E-cadherin expression. Their findings potentially suggest that antiestrogen therapy can result in restoration of E-cadherin expression.

Finally, regarding the role of BRCA in breast cancers, it has been reported that most of the tumors with BRCA-1 mutation are ER-negative and grade $3 .^{8}$ In the present series, several tumors $(32 \%)$ displayed loss of reactivity for BRCA-1 protein and had grade 3 histology (94\%). Robson et al have also suggested that BRCA-associated breast cancers were less likely to present with stage I disease, and more likely to have grade 3 tumors and axillary nodal metastases, compared to cases without mutations. Estrogen receptor negativity was also significantly more in BRCA-associated breast cancers (70 vs $34 \%$, $P=0.04)$.

In summary, ER-negative breast cancers are a distinct group of tumors with several unique morphological features. High grade, pushing margin, lymphoid stroma, comedo-type necrosis and central fibrosis/necrosis are the dominant morphological findings. In addition, apocrine features, squamoid, clear cell and spindle cell differentiation may be identified. Several distinct subtypes such as metaplastic carcinomas, adenoid cystic carcinoma, apocrine carcinoma and acinic cell carcinoma are present in this group.

The presence of pushing margin appears to have a significant correlation with negative lymph node status. The majority of these lesions are grade 3 carcinomas, a finding that correlates with the presence of lymphoid stroma and comedo-type necrosis. Alterations in protein expression of p53 and BRCA-1 and overexpression of Her2/neu and EGFR are also more commonly seen than in the whole population of invasive breast cancers. The above factors, in combination with worse tumor grade contribute to the aggressive biologic behavior of ER-negative tumors.

\section{References}

1 Maynard PV, Davis CJ, Blamer RW, et al. Relationship between estrogen-receptor content and histologic grade in human primary breast tumors. Br J Cancer 1978; 38:745-748.

2 Parl FF, Schmidt BP, Dupont WD, et al. Prognostic significance of estrogen receptor status in breast cancer in relation to tumor stage, axillary node metastasis, and histopathologic grading. Cancer 1984;54:2237-2242.

3 Kinne DW, Butler JA, Kimmel M, et al. Estrogen receptor protein of breast cancer in patients with positive nodes. High recurrence rates in the postme- nopausal estrogen receptor-negative group. Arch Surg 1987;122:1303-1306.

4 Pichon MF, Broet P, Magdelenat $\mathrm{H}$, et al. Prognostic value of steroid receptors after long-term follow-up of 2257 operable breast cancers. Br J Cancer 1996;73: 1545-1551.

5 Trendell-Smith NJ, Peston D, Shousha S. Adenoid cystic carcinoma of the breast: a tumour commonly devoid of oestrogen receptors and related proteins. Histopathology 1999;35:241-248.

6 Rosen PP, Cranor ML. Secretory carcinoma of the breast. Arch Pathol Lab Med 1991;115:141-144.

7 Jensen ML, Kiaer H, Andersen J, et al. Prognostic comparison of three classifications for medullary carcinomas of the breast. Histopathology 1997;30: $523-532$

8 Robson M, Gilewski T, Haas B, et al. BRCA-associated breast cancer in young women. J Clin Oncol 1998;16: 1642-1649.

9 Ellis IO, Galea M, Broughton N, et al. Pathological prognostic factors in breast cancer. II. Histological type. Relationship with survival in a large study with long-term follow-up. Histopathology 1992;20: 479-489.

10 Elston CW, Ellis IO. Pathological prognostic factors in breast cancer. I. The value of histological grade in breast cancer: experience from a large study with long term follow up. Histopathology 1991;19:403-410.

11 Galea MH, Blamey RW, Elston CE, et al. The Nottingham Prognostic Index in primary breast cancer. Breast Cancer Res Treat 1992;22:207-219.

12 Kononen J, Bubendorf L, Kallioniemi A, et al. Tissue microarrays for high-throughput molecular profiling of tumor specimens. Nat Med 1998;4:844-847.

13 McCarty Jr KS, McCarty Sr KS. Histochemical approaches to steroid receptor analyses. Semin Diagn Pathol 1984;1:297-308.

14 Peto R, Boreham J, Clarke M, et al. UK and USA breast cancer deaths down $25 \%$ in year 2000 at ages 20-69 years. Lancet 2000;355:1822.

15 Fisher B, Redmond C, Fisher ER, et al. Relative worth of estrogen or progesterone receptor and pathologic characteristics of differentiation as indicators of prognosis in node negative breast cancer patients: findings from National Surgical Adjuvant Breast and Bowel Project Protocol B-06. J Clin Oncol 1988;6: 1076-1087.

16 Pia-Foschini M, Reis-Filho JS, Eusebi V, et al. Salivary gland-like tumours of the breast: Surgical and molecular pathology. J Clin Pathol 2003;56:497-506.

17 Holst VA, Marshall CE, Moskaluk CA, et al. KIT protein expression and analysis of c-kit gene mutation in adenoid cystic carcinoma. Mod Pathol 1999;12: 956-960.

18 Scawn R, Shousha S. Morphologic spectrum of estrogen receptor-negative breast carcinoma. Arch Pathol Lab Med 2002;126:325-330.

19 Allred DC, Harvey JM, Berardo M, et al. Prognostic and predictive factors in breast cancer by immunohistochemical analysis. Mod Pathol 1998;11:155-168.

20 Iwao K, Matoba R, Ueno N, et al. Molecular classification of primary breast tumors possessing distinct prognostic properties. Hum Mol Genet 2002;11: 199-206.

21 Nixon AJ, Schnitt SJ, Gelman R, et al. Relationship of tumor grade to other pathologic features and to treatment outcome of patients with early stage breast 
carcinoma treated with breast-conserving therapy. Cancer 1996;78:1426-1431.

22 Rosen PP. Rosen's Breast Pathology, 2nd edn. Lippincott Williams and Wilkins: Philadelphia, 2001, pp 341.

23 Pupa SM, Bufalino R, Invernizzi AM, et al. Macrophage infiltrate and prognosis in c-erbB-2-overexpressing breast carcinomas. J Clin Oncol 1996;14:85-94.

24 Carter D, Pipkin RD, Shepard RH, et al. Relationship of necrosis and tumor border to lymph node metastases and 10-year survival in carcinoma of the breast. Am J Surg Pathol 1978;2:39-46.

25 Gilchrist KW, Gray R, Fowble B, et al. Tumor necrosis is a prognostic predictor for early recurrence and death in lymph node-positive breast cancer: a 10-year followup study of 728 Eastern Cooperative Oncology Group patients. J Clin Oncol 1993;11:1929-1935.

26 Jimenez RE, Wallis T, Visscher DW. Centrally necrotizing carcinomas of the breast: a distinct histologic subtype with aggressive clinical behavior. Am J Surg Pathol 2001;25:331-337.

27 Tsuda H, Takarabe T, Hasegawa T, et al. Myoepithelial differentiation in high-grade invasive ductal carcinomas with large central acellular zones. Hum Pathol 1999;30:1134-1139.

28 Colpaert C, Vermeulen P, van Beest P, et al. Intratumoral hypoxia resulting in the presence of a fibrotic focus is an independent predictor of early distant relapse in lymph node-negative breast cancer patients. Histopathology 2001;39:416-425.

29 Hasebe T, Sasaki S, Imoto S, et al. Prognostic significance of fibrotic focus in invasive ductal carcinoma of the breast: a prospective observational study. Mod Pathol 2002;15:502-516.

30 Pinder SE, Ellis IO, Galea M, et al. Pathological prognostic factors in breast cancer. III. Vascular invasion: relationship with recurrence and survival in a large study with long-term follow-up. Histopathology 1994;24:41-47.

31 Locker AP, Ellis IO, Morgan DA, et al. Factors influencing local recurrence after excision and radiotherapy for primary breast cancer. Br J Surg 1989;76:890-894.

32 Agoff SN, Swanson PE, Linden H, et al. Androgen receptor expression in estrogen receptor-negative breast cancer. Immunohistochemical, clinical and prognostic associations. Am J Clin Pathol 2003;120:725-731.

33 Gatalica Z. Immunohistochemical analysis of apocrine breast lesions. Consistent over-expression of androgen receptor accompanied by the loss of estrogen and progesterone receptors in apocrine metaplasia and apocrine carcinoma in situ. Pathol Res Pract 1997;193:753-758.

34 Sainsbury JR, Farndon JR, Needham GK, et al. Epidermal-growth-factor receptor status as predictor of early recurrence of and death from breast cancer Lancet 1987;1:1398-1402.

$35 \mathrm{Lu} \mathrm{C}$, Speers C, Zhang Y, et al. Effect of epidermal growth factor receptor inhibitor on development of estrogen receptor-negative mammary tumors. J Natl Cancer Inst 2003;95:1825-1833.

36 Horiguchi J, Koibuchi Y, Iijima K, et al. Immunohistochemical double staining with estrogen receptor and HER2 on primary breast cancer. Int J Mol Med 2003; 12:855-859.

37 Slamon DJ, Clark GM, Wong SG, et al. Human breast cancer: correlation of relapse and survival with amplification of the HER-2/neu oncogene. Science 1987;235:177-182.

38 Soomro S, Shousha S, Taylor P, et al. c-erbB-2 expression in different histological types of invasive breast carcinoma. J Clin Pathol 1991;44:211-214.

39 Somerville JE, Clarke LA, Biggart JD. C-erbB-2 expression and histological type of in situ and invasive breast carcinoma. J Clin Pathol 1992;45:16-20.

40 Pharoah PD, Day NE, Caldas C. Somatic mutations in the p53 gene and prognosis in breast cancer: a metaanalysis. Br J Cancer 1999;8:1968-1973.

41 Brotherick I, Robson CN, Browell DA, et al. Cytokeratin expression in breast cancer: phenotypic changes associated with disease progression. Cytometry 1998;32:301-308.

42 Malzahn K, Mitze M, Thoenes M, et al. Biological and prognostic significance of stratified epithelial cytokeratins in infiltrating ductal breast carcinomas. Virchows Arch 1998;433:119-129.

43 Moll R, Mitze M, Frixen UH, et al. Differential loss of E-cadherin expression in infiltrating ductal and lobular breast carcinomas. Am J Pathol 1993;143: 1731-1742.

44 Kowalski PJ, Rubin MA, Kleer CG. E-cadherin expression in primary carcinomas of the breast and its distant metastases. Breast Cancer Res 2003;5:R217-R222.

45 Oesterreich S, Deng W, Jiang S, et al. Estrogenmediated down-regulation of E-cadherin in breast cancer cells. Cancer Res 2003;63:5203-5208. 